\title{
Violation of thermal conductivity bound in Horndeski theory
}

\author{
Hai-Shan Liu ${ }^{*}$ \\ Institute for Advanced Physics and Mathematics, Zhejiang University of Technology, \\ Hangzhou 310023, China
}

(Received 3 May 2018; published 25 September 2018)

\begin{abstract}
We consider charged anti-de Sitter planar black holes in the four-dimensional Einstein-MaxwellHorndeski theory with two free axions and analyze the black hole thermodynamics. We calculate the holographic thermoelectric conductivities of the dual field theory and determine the ratio of thermal conductivity over the temperature. At low temperature and with zero electric current, we find that the ratio is proportional to temperature squared and hence can be arbitrarily small, providing the first example that violates the previously conjectured thermal conductivity bound.
\end{abstract}

DOI: 10.1103/PhysRevD.98.061902

\section{INTRODUCTION}

The gauge-gravity duality has brought many remarkable insights to the dynamics of some strongly coupled condensed matter systems [1-4]. Several universal bounds of transport coefficients, including the well-known bound of the ratio of the shear viscosity to the entropy density $[5,6]$, have been conjectured based on the holographic "bottom-up" models. However, many of these bounds were later violated through various ways $[7,8]$. One bound that stands out is that for the thermal conductivity $\kappa_{\mathrm{dc}}$, at zero electric current. This thermal bound was formulated by using a simple holographic model of Einstein-Maxwell-Dilaton theory, together with some momentum dissipation mechanism, which states that [9]

$$
\frac{\kappa_{\mathrm{dc}}}{T} \geq \mathcal{C}
$$

where $\mathcal{C}$ is a nonzero finite constant, as long as the dilaton potential is bounded from below. ${ }^{1}$ Remarkably, this bound has been tested positive against a variety of holographic models, and up until now, there is no counterexample.

Motivated by searching for an counterexample, we study the holographic thermoelectric properties of black holes in Horndeski models. The Horndeski theories were constructed in the 1970s [11], and they were rediscovered and

\footnotetext{
*hsliu.zju@gmail.com

${ }^{1}$ It is worth pointing out that for unbounded potential the constant $\mathcal{C}$ approaches $1 /\left(-V_{\min }\right)$, where $V_{\min }$ is the minimum of the dilaton potential and approaches $-\infty$, and thus $\kappa_{\mathrm{dc}} / T$ can be zero at low temperatures as is mentioned in [10].

Published by the American Physical Society under the terms of the Creative Commons Attribution 4.0 International license. Further distribution of this work must maintain attribution to the author(s) and the published article's title, journal citation, and DOI. Funded by SCOAP ${ }^{3}$.
}

have received much attention in cosmology [12]. A particular property of Horndeski theories is that although their Lagrangians contain terms which have more than two derivatives, the equations of motion involve at most two derivatives on each field. This is analogous to Lovelock gravities [13], and the theories can be ghost free.

Black hole solutions that are asymptotic to (locally) antide Sitter (AdS) spacetime have been constructed in Horndeski gravity theories in $[14,15]$ and the thermodynamics of these AdS black holes were analyzed in $[16,17]$. The stability and causality were studied in [18-20]. Holographic properties in Horndeski theories were deeply investigated in [21-25]. Further applications and properties were discussed in [26-28]. It turns out that for general coupling constants, there is no holographic $a$-theorem for Horndeski gravity; however, there exists a critical point, holographic $a$-theorem can be established [25]. This implies that holographic application for Horndeski gravity is only sensible at the critical point of the coupling constant, where the dual field theory is scale invariant, rather than fully conformal invariant [25].

In this paper, we shall consider $D=4$ Einstein-MaxwellHorndeski gravity with a bare cosmological constant, together with two free axions for momentum dissipation. In Sec. II, we obtain charged AdS black holes at the critical point of the coupling constants. In Sec. III, we analyze the black hole thermodynamics. We study the holographic dc electrothermal conductivities in Sec. IV and find a counterexample of the bound (1.1). We conclude the paper in Sec. V.

\section{THE THEORY AND AdS PLANAR BLACK HOLES}

The Lagrangian of Einstein-Maxwell-Horndeski theory with bare cosmological constant $\Lambda$ and two free axions $\phi_{i}$ is 


$$
\begin{aligned}
\mathcal{L}= & \sqrt{g}\left[\kappa\left(R-2 \Lambda-\frac{1}{4} F^{2}\right)-\frac{1}{2}\left(\alpha g^{\mu \nu}-\gamma G^{\mu \nu}\right) \partial_{\mu} \chi \partial_{\nu} \chi\right. \\
& \left.-\frac{1}{2} \sum_{i=1}^{2}\left(\partial \phi_{i}\right)^{2}\right]
\end{aligned}
$$

where $F=d A$ and $G_{\mu \nu}$ is the Einstein tensor, and $\gamma$ is the Horndeski coupling. The theory admits an AdS planar black hole solution [21]

$$
\begin{aligned}
d s^{2}= & -h(r) d t^{2}+\frac{d r^{2}}{f(r)}+r^{2} d x_{i} d x_{i}, \quad A=a(r) d t, \\
\chi= & \chi(r), \quad \phi_{1,2}=\lambda x_{1,2}, \\
h= & g^{2} r^{2}-\frac{2 \kappa \lambda^{2}}{\beta \gamma+4 \kappa}-\frac{m_{0}}{r}+\frac{\kappa\left(3 g^{2} q^{2}(\beta \gamma+4 \kappa)-\kappa \lambda^{4}\right)}{3 g^{2} r^{2}(\beta \gamma+4 \kappa)^{2}} \\
& -\frac{\kappa^{2} q^{4}}{60 g^{2} r^{6}(\beta \gamma+4 \kappa)^{2}}-\frac{\kappa^{2} \lambda^{2} q^{2}}{9 g^{2} r^{4}(\beta \gamma+4 \kappa)^{2}}, \\
f= & \frac{36 g^{4} r^{8}(\beta \gamma+4 \kappa)^{2}}{\left(6 g^{2} r^{4}(\beta \gamma+4 \kappa)-\kappa\left(q^{2}+2 \lambda^{2} r^{2}\right)\right)^{2}} h, \\
\chi^{\prime}= & \sqrt{\frac{6 \beta \gamma g^{2} r^{4}-\kappa\left(q^{2}+2 \lambda^{2} r^{2}\right)}{6 \gamma g^{2} r^{4}}} \frac{1}{f}, \\
a= & a_{0}-\frac{q}{r}+\frac{q \kappa \lambda^{2}}{9 g^{2} r^{3}(\beta \gamma+4 \kappa)}+\frac{\kappa q^{3}}{30 g^{2} r^{5}(\beta \gamma+4 \kappa)},
\end{aligned}
$$

at the critical point of the coupling constants

$$
\Lambda=-\frac{3 g^{2}(\beta \gamma+2 \kappa)}{2 \kappa}, \quad \alpha=3 \gamma g^{2} .
$$

The solution with $\lambda=0$ was constructed in [15]. The parameters $a_{0}, q, m_{0}$ are integration constants. When the parameters $\left(m_{0}, q, \lambda\right)$ all vanish, the solution becomes the planar AdS vacuum but with nonvanishing $\chi$, namely

$$
\chi=\chi_{0}+\sqrt{\beta} g^{-1} \log r .
$$

Thus the special conformal transformation of the AdS vacuum is not preserved by $\chi$, but the Poincare symmetry and the scale invariance survive, leading to a scale invariant quantum field theory [25]. It is important to note that at the critical condition (2.3) for the coupling constants, the Horndeski coupling $\gamma$ does not have zero limit; it is part of the vacuum construction analogous to the bare cosmological constant and should not be viewed as a perturbative parameter. In fact for large AdS radius $\ell=1 / g$ where the AdS/CFT correspondence is applicable, the critical condition (2.3) implies that the Horndeski coupling constant must be large, which is significantly different from the Lovelock theories, or the noncritical cases where $\gamma$ is fixed and can be arbitrarily small.
There is a curvature singularity located at $r=r_{*}$, where $f$ diverges, and it is determined by

$$
F\left(r_{*}\right) \equiv 6 g^{2} r_{*}^{4}(\beta \gamma+4 \kappa)-\kappa\left(q^{2}+2 \lambda^{2} r_{*}^{2}\right)=0 .
$$

In order to describe a black hole, we require that the largest root $r_{*}$ should be inside the event horizon, that is $r_{*}<r_{0}$, where the radius of the event horizon $r_{0}$ is the largest root of $f\left(r_{0}\right)=0$. The Hawking temperature can be obtained through standard method

$$
T=\frac{6 g^{2} r_{0}^{4}(\beta \gamma+4 \kappa)-\kappa q^{2}-2 r_{0}^{2} \kappa \lambda^{2}}{8 \pi r_{0}^{3}(\beta \gamma+4 \kappa)} .
$$

It is worthwhile pointing out that the requirement $r_{*}<r_{0}$ guarantees that the temperature is positive definite. In particular, the temperature can be arbitrarily close to zero, but cannot reach zero.

Though the linearized equations of motion for Horndeski theory involve only two derivatives, it is still necessary to insure that the kinetic term of the Horndeski scalar is positive to avoid any possible ghostlike behavior. The kinetic term for the axion perturbation $\delta \chi$ is $P^{00} \delta \dot{\chi} \delta \dot{\chi}$, where

$$
P^{00}=\frac{144 \gamma g^{4} \kappa r^{6}(\beta \gamma+4 \kappa)^{2}\left(q^{2}+\lambda^{2} r^{2}\right)}{F(r)^{3}} .
$$

Here $F(r)$ is defined in (2.5). In order to avoid any ghostlike excitation, $P^{00}$ should be non-negative from horizon to asymptotic infinity. As was discussed above, the requirement $r_{*}<r_{0}$ implies that $F(r)$ is always positive on and outside the horizon. It follows that the positivity of $P^{00}$ requires that $\gamma>0$, which is consistent with the critical condition (2.3).

\section{BLACK HOLE THERMODYNAMICS}

The thermodynamics of an AdS black hole in Horndeski theory have been studied extensively in $[16,17]$ with the aid of Wald formalism [29,30]. Here, we begin by reviewing the main results. The variation of Lagrangian $\mathcal{L}$ gives the equations of motion (EOM) and total derivative terms

$$
\delta \mathcal{L}=\mathrm{EOM}+\sqrt{g} \nabla_{\mu} J^{\mu},
$$

from which we can define a 1 -form $J_{(1)}=J_{\mu} d x^{\mu}$ and its Hodge dual $\Theta_{(3)}=-1 * J_{(1)}$. Specializing the variation to be induced by an infinitesimal diffeomorphism $\delta x^{\mu}=\xi^{\mu}$, we can define a 3 -form and show that

$$
J_{(3)} \equiv \Theta_{(3)}-i_{\xi} * \mathcal{L}=\mathrm{EOM}-d * J_{(2)},
$$

where $i_{\xi}$ represents a contraction of $\xi^{\mu}$ with the 3 -form $* \mathcal{L}$ and $J_{(2)}=d \xi$. Then, one can define a 2-form $Q_{(2)} \equiv * J_{(2)}$, such that $J_{(3)}=d Q_{(2)}$ on shell. The variation of the Hamiltonian is given by 


$$
\begin{aligned}
\delta H= & \delta Q-i_{\xi} \Theta=-2 r \sqrt{\frac{h}{f}}\left(\kappa+\frac{\gamma}{4} f \chi^{\prime 2}\right) \delta f \Omega_{(2)} \\
& -r^{2} \sqrt{\frac{h}{f}} \kappa\left(\frac{f}{h} a \delta a^{\prime}+\frac{a a^{\prime}}{2}\left(\frac{\delta f}{h}-\frac{f \delta h}{h^{2}}\right)\right) \Omega_{(2)} .
\end{aligned}
$$

Note that here we consider $\lambda$ and $g=1 / \ell$ as thermodynamical constants. We also choose a gauge that the electric potential vanishes on the horizon, then the variation of the Hamiltonian on the horizon is

$$
\delta H_{+}=16 \pi T\left(\kappa+\frac{\gamma}{4} f \chi^{\prime 2}\right) \delta\left(\frac{r_{0}^{2}}{4}\right)
$$

where $T$ is given in (2.6), while at the infinity, it is

$$
\delta H_{\infty}=\kappa \mu \delta q-\frac{(4 \kappa+\beta \gamma) \delta m_{0}}{2}
$$

and Wald showed that the variation of the Hamiltonian vanishes on the Cauchy surface. For a black hole it implies that $\delta H_{+}+\delta H_{\infty}=0$, which gives the first law of thermodynamics,

$$
d M=T d S+\Phi_{e} d Q_{e}+\Phi_{\chi}^{+} d Q_{\chi}^{+}
$$

with

$$
\begin{aligned}
M & =\frac{1}{2}(4 \kappa+\beta \gamma) m_{0}, \quad \Phi_{e}=\mu, \quad Q_{e}=\kappa q, \\
S & =\left(\kappa+\left.\frac{\gamma}{4}\left(f \chi^{\prime 2}\right)\right|_{r_{0}}\right) 4 \pi r_{0}^{2}=\frac{16 \pi r_{0}(\beta \gamma+4 \kappa)}{3 g^{2}} T, \\
\Phi_{\chi}^{+} & =-\frac{\gamma r_{0}^{2} T}{8} \sqrt{\left.f \chi^{\prime 2}\right|_{r_{0}}}, \\
Q_{\chi}^{+} & =16 \pi \int_{r=r_{0}} \sqrt{(\partial \chi)_{+}^{2}}=\left.16 \pi \sqrt{f} \chi^{\prime}\right|_{r=r_{0}} .
\end{aligned}
$$

$\Phi_{\chi}^{+}$and $Q_{\chi}^{+}$are the scalar potential and charge, respectively (see [17] for more details). The temperature is given in (2.6). It is important to note that owing to our parametrization we appear to be able to set $\gamma=0$ in the above thermodynamical quantities; however, as we have remarked earlier, there is no smooth limit of $\gamma=0$.

\section{HOLOGRAPHIC THERMOELECTRIC CONDUCTIVITY}

There are various ways to obtain the holographic dc thermoelectric conductivities [31-34]. The key step is to construct the relevant radially conserved current, which serves as a bridge connecting the boundary physical properties to the black hole horizon information. We consider the following perturbations around the background solution,

$$
\begin{array}{ll}
\delta g_{t x_{1}}=t U_{1}(r)+\Psi_{t x_{1}}, & \delta g_{r x_{1}}=\Psi_{r x_{1}}, \\
\delta A_{x_{1}}=t U_{2}(r)+a_{x_{1}}, & \delta \phi_{1}=\frac{\Phi(r)}{\lambda} .
\end{array}
$$

The radially conserved electric current can be easily obtained with the help of Maxwell equation $\partial_{r}\left(\sqrt{g} F^{r x_{1}}\right)=0$,

$$
\mathcal{J}=\kappa \sqrt{g} F^{r x_{1}}
$$

The radially conserved holographic heat current is more difficult to construct, since its conservation involves both Einstein and Maxwell equations. Fortunately, a general formula of deriving the holographic heat current was proposed in [24] by using Noether symmetry for general classes of gravity theories. Applying this formula in our theory, we obtain the holographic heat current

$$
\mathcal{Q}=\sqrt{g}\left(\kappa\left(2 \nabla^{r} \xi^{x_{1}}+a F^{r x_{1}}\right)+\frac{\gamma}{2} g^{r r}\left(\partial_{r} \chi\right)^{2} \nabla^{r} \xi^{x_{1}}\right),
$$

where $\xi$ is the timelike Killing vector $\partial_{t}$. We find that the electric and heat current can be time independent by choosing

$$
U_{1}=-\zeta h, \quad U_{2}=-E+\zeta a,
$$

where $E$ and $\zeta$ are constants which parametrize the sources for the electric and heat currents, respectively. Near the black hole horizon, we impose the ingoing wave condition

$a_{x_{1}}^{\prime}=\frac{-E+\zeta a}{\sqrt{h f}}+\cdots, \quad \Psi_{t x_{1}}=\Psi_{t x_{1}}^{(0)}-\zeta h \int \frac{1}{\sqrt{h f}}+\cdots$,

where $\Psi_{t x_{1}}^{(0)}$ is a regular function whose value on the horizon can be determined by the linearized perturbative equation of motion

$$
\Psi_{t x_{1}}^{(0)}\left(r_{0}\right)=-\frac{48 E g^{2} \kappa q r_{0}^{5}(\beta \gamma+4 \kappa)+\zeta\left(\kappa\left(q^{2}+2 \lambda^{2} r_{0}^{2}\right)-6 g^{2} r^{4}(\beta \gamma+4 \kappa)\right)^{2}}{48 g^{2} \kappa \lambda^{2} r_{0}^{5}(\beta \gamma+4 \kappa)}
$$

Now, we are in a position to evaluate the radially conserved currents on the horizon 
$\mathcal{J}=\left(\kappa+\frac{\kappa q^{2}}{\lambda^{2} r_{0}^{2}}\right) E+\frac{4 \pi^{2} q(\beta \gamma+4 \kappa)}{3 g^{2} \lambda^{2} r_{0}} T^{2} \zeta$

$\mathcal{Q}=\frac{4 \pi^{2} q(\beta \gamma+4 \kappa)}{3 g^{2} \lambda^{2} r} T^{2} E+\frac{16 \pi^{4}(\beta \gamma+4 \kappa)^{2}}{9 g^{4} \kappa \lambda^{2}} T^{4} \zeta$.

The dc conductivity matrix is then given by

$$
\begin{aligned}
\sigma_{\mathrm{dc}} & =\frac{\partial \mathcal{J}}{\partial E}=\kappa\left(1+\frac{q^{2}}{r_{0}^{2} \lambda^{2}}\right), \\
\alpha_{\mathrm{dc}} & =\frac{1}{T} \frac{\partial \mathcal{J}}{\partial \zeta}=\frac{4 \pi^{2} q(\beta \gamma+4 \kappa)}{3 g^{2} r_{0} \lambda^{2}} T, \\
\bar{\alpha}_{\mathrm{dc}} & =\frac{1}{T} \frac{\partial \mathcal{Q}}{\partial E}=\frac{4 \pi^{2} q(\beta \gamma+4 \kappa)}{3 g^{2} r_{0} \lambda^{2}} T, \\
\bar{\kappa}_{\mathrm{dc}} & =\frac{1}{T} \frac{\partial \mathcal{Q}}{\partial \zeta}=\frac{16 \pi^{4}(\beta \gamma+4 \kappa)^{2}}{9 \kappa g^{4} \lambda^{2}} T^{3} .
\end{aligned}
$$

It is thus clear that the electric bound, which was proposed in [35], is satisfied

$$
\sigma_{\mathrm{dc}}=\kappa\left(1+\frac{q^{2}}{r_{0}^{2} \lambda^{2}}\right) \geq 1
$$

The form of the electric conductivity $\sigma_{\mathrm{dc}}$ is the same as that of the Einstein-Maxwell case [36] while it is expressed in terms of black hole horizon radius $r_{0}$, as is pointed out in [21]. It is easy to check that $\alpha_{\mathrm{dc}}=\bar{\alpha}_{\mathrm{dc}}$, which means the Onsager relation holds. Furthermore, we also find that the thermal relation $S T \alpha_{\mathrm{dc}}-Q_{e} \bar{\kappa}_{\mathrm{dc}}=0$ holds for this system.

The thermal conductivity at zero electric current is

$$
\kappa_{\mathrm{dc}}=\frac{16 \pi^{4}(\beta \gamma+4 \kappa)^{2}}{9 \kappa g^{4}\left(\lambda^{2}+\frac{q^{2}}{r_{0}^{2}}\right)} T^{3}
$$

There are two more quantities of interest, the Lorentz ratios of the thermal conductivities over the electric conductivities, which are given by

$\bar{L}=\frac{\bar{\kappa}_{\mathrm{dc}}}{\sigma_{\mathrm{dc}} T}=\frac{S^{2}}{\kappa^{2}\left(q^{2}+\lambda^{2} r_{0}^{2}\right)}, \quad L=\frac{\kappa_{\mathrm{dc}}}{\sigma_{\mathrm{dc}} T}=\frac{\lambda^{2} r_{0}^{2} S^{2}}{\kappa^{2}\left(q^{2}+\lambda^{2} r_{0}^{2}\right)^{2}}$

Usually, the Lorentz ratio $L$ is a constant, due to the fact that the heat transport and the electric transport both involve the charge carriers, like free electrons in metal, which is well known as the Wiedemann-Franz law. As we can see, this law is violated in our case, which may be explained in terms of independent transportation of charge and heat in a strongly coupled system.

It was observed that there is a bound for Lorentz ratio $\bar{L}$ in [34]

$$
\bar{L} \leq \frac{S^{2}}{Q_{e}^{2}} .
$$

From (4.11), we can see this bound is indeed satisfied in our case

$$
\bar{L}=\frac{S^{2}}{\kappa^{2}\left(q^{2}+\lambda^{2} r_{0}^{2}\right)} \leq \frac{S^{2}}{Q_{e}^{2}} .
$$

Having established that both the electric bound (4.9) and the Lorentz ratio bound (4.13) are satisfied, we now examine the thermal conductivity bound (1.1). As follows from (4.10), we find

$$
\frac{\kappa_{\mathrm{dc}}}{T}=\frac{16 \pi^{4}(\beta \gamma+4 \kappa)^{2}}{9 \kappa g^{4}\left(\lambda^{2}+\frac{q^{2}}{r_{0}^{2}}\right)} T^{2} .
$$

As discussed in Sec. II, although the temperature of the black hole cannot be zero, it can be arbitrarily close to zero. For sufficiently low temperature, the ratio becomes

$\frac{\kappa_{\mathrm{dc}}}{T} \sim \frac{16 \pi^{4}(\beta \gamma+4 \kappa)^{2}}{9 g^{4} \sqrt{\kappa} \sqrt{6 g^{2} q^{2}(\beta \gamma+4 \kappa)+\kappa \lambda^{4}}} T^{2}+\mathcal{O}\left(T^{3}\right)$.

Thus, the ratio can be arbitrarily small at low temperature and it is obvious that the scalar potential of Horndeski theory is bounded, hence our holographic model violates the proposed thermal conductivity bound (1.1).

\section{CONCLUSIONS}

In this paper, we considered the Einstein-MaxwellHorndeski theory with bare cosmological constant and two free axions. The theory admits analytical charged AdS planar black holes, where the axions span over the twodimensional plane. It is important to note that the AdS spacetime where the black holes are immersed in is the vacuum solution at the critical point of the coupling constants. The special conformal transformation of the $\mathrm{AdS}$ is broken by the Horndeski scalar $\chi$, but the Poincaré and scale invariance survive, giving rise to scale invariant quantum field theory at the boundary. We analyzed the thermodynamics of the black hole and calculated the holographic thermoelectric conductivities of the dual field theory. The focus of the paper is to examine various related universal holographic bounds proposed in literature.

The Horndeski term does not contribute directly to the electric conductivity, which takes the same form as that of the Einstein-Maxwell theory. Thus the electric conductivity bound is preserved. Furthermore, the Onsager relation $\alpha_{\mathrm{dc}}=\bar{\alpha}_{\mathrm{dc}}$ and the thermal relation $S T \alpha_{\mathrm{dc}}-Q_{e} \bar{\kappa}_{\mathrm{dc}}=0$ are both satisfied. The situation for the thermal conductivity, on the other hand, is quite different. Although the Lorentz ratio bound is satisfied, the ratio of thermal conductivity at zero electric current over temperature turns out to be, at low temperature, constantly proportional to the square of the temperature; therefore, it can be arbitrarily small as the temperature is low, violating the conductivity bound (1.1). This rare counterexample indicates that an underlying principal is needed to understand the condition when the bound is valid. It is of interest to investigate whether the breaking of the conformal symmetry to sale invariance is the culprit for the bound violation. 


\section{ACKNOWLEDGMENTS}

We thank the anonymous referees for their useful suggestions which improve the paper a lot. H.-S. L. is supported in part by NSFC Grants No. 11305140, No. 11375153, No. 11475148, and No. 11675144.

[1] S. A. Hartnoll, Lectures on holographic methods for condensed matter physics, Classical Quantum Gravity 26, 224002 (2009).

[2] S. Sachdev, What can gauge-gravity duality teach us about condensed matter physics?, Annu. Rev. Condens. Matter Phys. 3, 9 (2012).

[3] J. McGreevy, TASI lectures on quantum matter (with a view toward holographic duality), arXiv:1606.08953.

[4] J. Zaanen, Y. W. Sun, Y. Liu, and K. Schalm, Holographic Duality in Condensed Matter Physics (Cambridge Univ. Press, Cambridge, England, 2015).

[5] P. Kovtun, D. T. Son, and A. O. Starinets, Holography and hydrodynamics: Diffusion on stretched horizons, J. High Energy Phys. 10 (2003) 064.

[6] P. Kovtun, D. T. Son, and A. O. Starinets, Viscosity in Strongly Interacting Quantum Field Theories from Black Hole Physics, Phys. Rev. Lett. 94, 111601 (2005).

[7] M. Baggioli and O. Pujolas, On holographic disorder-driven metal-insulator transitions, J. High Energy Phys. 01 (2017) 040 .

[8] B. Goutéraux, E. Kiritsis, and W. J. Li, Effective holographic theories of momentum relaxation and violation of conductivity bound, J. High Energy Phys. 04 (2016) 122.

[9] S. Grozdanov, A. Lucas, and K. Schalm, Incoherent thermal transport from dirty black holes, Phys. Rev. D 93, 061901 (2016).

[10] M. Blake, Universal diffusion in incoherent black holes, Phys. Rev. D 94, 086014 (2016).

[11] G. W. Horndeski, Second-order scalar-tensor field equations in a four-dimensional space, Int. J. Theor. Phys. 10, 363 (1974).

[12] A. Nicolis, R. Rattazzi, and E. Trincherini, The Galileon as a local modification of gravity, Phys. Rev. D 79, 064036 (2009).

[13] D. Lovelock, The Einstein tensor and its generalizations, J. Math. Phys. (N.Y.) 12, 498 (1971).

[14] A. Anabalon, A. Cisterna, and J. Oliva, Asymptotically locally AdS and flat black holes in Horndeski theory, Phys. Rev. D 89, 084050 (2014).

[15] A. Cisterna and C. Erices, Asymptotically locally AdS and flat black holes in the presence of an electric field in the Horndeski scenario, Phys. Rev. D 89, 084038 (2014).

[16] X. H. Feng, H. S. Liu, H. Lü, and C. N. Pope, Black hole entropy and viscosity bound in Horndeski gravity, J. High Energy Phys. 11 (2015) 176.

[17] X. H. Feng, H. S. Liu, H. Lü, and C. N. Pope, Thermodynamics of charged black holes in Einstein-HorndeskiMaxwell theory, Phys. Rev. D 93, 044030 (2016).

[18] J. B. Jimenez, R. Durrer, L. Heisenberg, and M. Thorsrud, Stability of Horndeski vector-tensor interactions, J. Cosmol. Astropart. Phys. 10 (2013) 064.
[19] T. Kobayashi, H. Motohashi, and T. Suyama, Black hole perturbation in the most general scalar-tensor theory with second-order field equations. II. The even-parity sector, Phys. Rev. D 89, 084042 (2014).

[20] M. Minamitsuji, Causal structure in the scalar-tensor theory with field derivative coupling to the Einstein tensor, Phys. Lett. B 743, 272 (2015).

[21] X. M. Kuang and E. Papantonopoulos, Building a holographic superconductor with a scalar field coupled kinematically to Einstein tensor, J. High Energy Phys. 08 (2016) 161.

[22] W. J. Jiang, H. S. Liu, H. Lü, and C. N. Pope, DC conductivities with momentum dissipation in Horndeski theories, J. High Energy Phys. 07 (2017) 084.

[23] M. Baggioli and W. J. Li, Diffusivities bounds and chaos in holographic Horndeski theories, J. High Energy Phys. 07 (2017) 055.

[24] H. S. Liu, H. Lü, and C. N. Pope, Holographic heat current as Noether current, J. High Energy Phys. 09 (2017) 146.

[25] Y.Z. Li and H. Lü, $a$-theorem for Horndeski gravity at the critical point, Phys. Rev. D 97, 126008 (2018).

[26] X. H. Feng, H. S. Liu, W. T. Lu, and H. Lü, Horndeski gravity and the violation of reverse isoperimetric inequality, Eur. Phys. J. C 77, 790 (2017).

[27] E. Caceres, R. Mohan, and P. H. Nguyen, On holographic entanglement entropy of Horndeski black holes, J. High Energy Phys. 10 (2017) 145.

[28] W. J. Geng, S. L. Li, H. Lü, and H. Wei, Godel metrics with chronology protection in Horndeski gravities, Phys. Lett. B 780, 196 (2018).

[29] R. M. Wald, Black hole entropy is the Noether charge, Phys. Rev. D 48, R3427 (1993).

[30] V. Iyer and R. M. Wald, Some properties of Noether charge and a proposal for dynamical black hole entropy, Phys. Rev. D 50, 846 (1994).

[31] N. Iqbal and H. Liu, Universality of the hydrodynamic limit in AdS/CFT and the membrane paradigm, Phys. Rev. D 79, 025023 (2009).

[32] M. Blake and D. Tong, Universal resistivity from holographic massive gravity, Phys. Rev. D 88, 106004 (2013).

[33] A. Donos and J. P. Gauntlett, Novel metals and insulators from holography, J. High Energy Phys. 06 (2014) 007.

[34] A. Donos and J. P. Gauntlett, Thermoelectric DC conductivities from black hole horizons, J. High Energy Phys. 11 (2014) 081.

[35] S. Grozdanov, A. Lucas, S. Sachdev, and K. Schalm, Absence of Disorder-Driven Metal-Insulator Transitions in Simple Holographic Models, Phys. Rev. Lett. 115, 221601 (2015).

[36] T. Andrade and B. Withers, A simple holographic model of momentum relaxation, J. High Energy Phys. 05 (2014) 101. 